\title{
Ultrathin and High-Efficiency Pancharatnam-Berry Phase Metalens for Millimeter Waves
}

\author{
Alexia Moreno-Peñarrubia ${ }^{1,2}$, Jorge Teniente ${ }^{1,2}$, Sergei Kuznetsov ${ }^{3,4}$, Bakhtiyar \\ Orazbayev $^{5}$ and Miguel Beruete ${ }^{1,2}$ \\ ${ }^{1}$ Dept. Electrical, Electronic and Communication Engineering, Universidad Pública de Navarra, 31006 Pamplona, Navarra, Spain \\ ${ }^{2}$ Institute of Smart Cities (ISC), Public University of Navarre, Campus de Arrosadia, 31006, Pamplona, Navarra, Spain \\ ${ }^{3}$ Rzhanov Institute of Semiconductor Physics SB RAS, Novosibirsk Branch' TDIAM', 630090, Novosibirsk, Russia \\ ${ }^{4}$ Analytical and Technological Research Center, Novosibirsk State University, 630090, Novosibirsk, Russia \\ ${ }^{5}$ Department of Physics, School of Sciences and Humanities, Nazarbayev University, 010000, Kazakhstan
}

Corresponding author: miguel.beruete@unavarra.es

Applying the Pancharatnam Berry (PB) principle to half-wave plate (HWP) metasurfaces allows the manipulation of wavefronts along with the conversion of the handedness of circularly polarized incident waves by simply rotating the metaatoms that compose the metasurface. PB metasurfaces (PBM) working in transmission mode with four or more layers have demonstrated to reach levels of transmission efficiency near $100 \%$ but also have resulted in bulky structures. On the other hand, compact tri-layer ultrathin $(\lambda / 8)$ designs have reached levels near $90 \%$ but are more challenging than single- or bi-layer structures from a manufacturing viewpoint. Here, we propose a compact ultrathin $(<\lambda / 13)$ transmissive PBM with only two layers (which significantly simplifies the fabrication process) achieving a transmission efficiency level of around $90 \%$, focusing the wavefront of a circularly polarized incident wave and converting its handedness. The metasurface is composed of identical bi-layered $\mathrm{H}$-shaped unit cells (meta-atoms) whose transmission phases are chosen by introducing different rotation angles to each unit cell according to a lens spatial phase profile. The structure is analytically and numerically studied and experimentally measured, verifying an excellent behavior as HWP PB metalens at $87 \mathrm{GHz}$.

Metasurfaces (also known as 2D metamaterials ${ }^{1-3}$ ) are planar structures engineered to control the waves in unconventional ways using subwavelength elements, called meta-atoms. Several metasurfaces have been reported using Half-Wave Plate (HWP) $)^{4,5}$ elements, where the latter converts the handedness of circularly polarized $(\mathrm{CP})$ incident waves. Such HWP gradient index (GRIN) metasurfaces ${ }^{6,7}$ are composed of different geometric meta-atoms to control wavefront shapes and simultaneously convert the polarization state. Interestingly, by applying the Pancharatnam Berry (PB) principle ${ }^{8}$ to HWP metasurfaces, multiple structures enabling the manipulation of wavefronts using rotated identical meta-atoms have been demonstrated ${ }^{9}$ ${ }^{11}$. In these devices, known as Pancharatnam-Berry metasurfaces (PBM), the spatial phase profile is engineered by the meta-atoms' rotation angle following the $\mathrm{PB}$ principle. According to this principle, when a $\mathrm{CP}$ wave crosses an HWP's unit cell rotated by an angle $\theta$, it acquires an additional phase shift of $2 \theta$ at the output.

Since then, such thin metasurface devices have been widely demonstrated to manipulate wavefronts using planar designs. As a result, several PBM devices operating even at optical frequencies ${ }^{12}$ have been presented, with metalenses reaching high focusing efficiencies up to $86 \%$. There are also examples of PBM working at the microwave or terahertz $(\mathrm{THz})$ range for functionalities such as beam steering ${ }^{13}$, lensing $^{14,15}$, vortex generation ${ }^{16-18}$, meta-couplers ${ }^{19}$. However, although PBMs have shown strong capabilities to control CP waves in reflection mode ${ }^{20}$, they are less efficient in transmission mode, limiting their applications in practice. Nevertheless, some PBMs operating in transmission mode have been demonstrated with efficiencies about $60 \%{ }^{21}$ or even near $100 \%$ by using multi-layer designs (four cascaded metasurfaces $)^{22}$. Unfortunately, these devices result in bulky structures $(\sim 4 \lambda / 3)$ and a significantly more complicated fabrication process, limiting their potential practical applications.

Lately, tri-layer PBM designs have been developed, obtaining very compact structures for controlling wavefronts. Recent examples of the ultrathin metalenses have achieved nearly $68 \%$ of the cross-polar transmission efficiency (with a thickness $\sim \lambda / 6)^{15}$, or even $90 \%$ of cross-polar transmission efficiency (with a thickness $\sim \lambda / 5)^{14}$. Moreover, a vortexbeam generator with $91 \%$ efficiency and thickness $\sim \lambda / 8$ has also been demonstrated ${ }^{18}$.

Despite having good efficiency and compactness, trilayer devices are more challenging than single- or bi-layer structures from a manufacturing viewpoint. Tri-layer designs require two substrate layers that entail strict alignment constraints and, more importantly, tight contact between them to avoid thin air layers that can ruin the performance. Single-layer devices operating in transmission mode can only reach $25 \%$ of cross-polar transmission efficiency ${ }^{23,24}$. Therefore, bi-layer PBM structures stand out as the preferred candidates for leveraging the fabrication constraints. However, it is challenging to design bi-layer devices with similar efficiency levels as tri-layer structures due to the reduced freedom to fine-tune the whole device's impedance 
matching. In addition, a reduced number of layers leads to a reduced number of poles in the device's transfer function and, therefore, in a narrower operating bandwidth than multi-layer designs.

Nevertheless, there are experimental and numerical demonstrations of bi-layer PBMs designs in the literature: a vortex generator that achieves a transmission efficiency of $55 \%$ (with a thickness $\lambda / 20)^{17}$, a beam steerer with a transmission efficiency of $80 \%$ (thickness below $\lambda / 10)^{13}$, and recently, a PB metalens with a cross-polar transmission efficiency of $82.7 \%$ (thickness below $\lambda / 8^{25}$ ). Although all these works achieve very high-efficiency values, all of them are below the best tri-layer designs ${ }^{18}$. To tackle the problem of efficiency, in this work, we experimentally demonstrate an ultrathin bi-layer PB metalens with a transmission efficiency of $90.15 \%$ and a thickness $<\lambda / 13$. The numerical analysis and measurements confirmed the device's ability to convert a $\mathrm{CP}$ wave's handedness and focus the wavefront at $94 \mathrm{~mm}$ from the metasurface at $87 \mathrm{GHz}$.

This paper is organized as follows: In the first part, we discuss the wave polarization control using the dipolar resonances of the metallic elements of the metasurface by analyzing the transmission and cross-polarization efficiencies. In the second part, we analyze the PBM metalens' focusing performance using a semi-analytical Huygens-Fresnel (H-F) analysis and full-wave simulations. Finally, the metalens' superior performance is experimentally verified using a circularly polarized incident wave and subsequent linear to circular basis transformation.

The PBM's unit cell used in this work consists of two aluminum layers of $\mathrm{H}$-shaped elements with a thickness of $0.55 \mu \mathrm{m}$ patterned on both faces of a thin polypropylene (PP) slab with a thickness of $254 \pm 5 \mu \mathrm{m}$ and dielectric permittivity of 2.25. A schematic with dimensions and a photograph of a unit cell are shown in Fig. 1(a) and (b), respectively. A PP slab was chosen as the substrate material due to its low dielectric losses $\left(\tan \delta \approx 1 \times 10^{-3}\right)^{11}$. Before micropatterning, the PP film was metalized from both sides via thermal deposition of aluminum in a vacuum. A contact photolithography technique ${ }^{26}$ was applied afterwards to create the $\mathrm{H}$-shaped patterns sequentially on the PP faces. Regarding the numerical characterization, all the simulations were performed using the commercial software CST Studio Suite $^{\circledR}$. The aluminum conductivity was taken as $2.7 \times 10^{7} \mathrm{~S} / \mathrm{m}$, smaller than the DC nominal value, to account for extra losses introduced by surface roughness.

First, we performed a detailed unit-cell study under linear polarization excitation ( $x$ - and $y$-components) to visualize the working principle of the HWP metasurface. We used the frequency domain solver to study the unit cell numerically with Floquet periodic boundary conditions using linear vertical ( $y$ electric field component) and horizontal ( $x$ electric field component) polarization excitation. The unit cell periodicity was fixed to $0.4 \lambda$, to avoid the onset of diffraction lobes which is at $107.14 \mathrm{GHz}$.

The transmission coefficient magnitudes $T_{x x}$ and $T_{y y}$ for one (dashed red and blue lines respectively) and two layers (solid red and blue lines respectively), and the phase difference between them (solid black line for two layers) as a function of frequency are shown in Fig. 2(a). A single layer under horizontal excitation has a dip at $58.4 \mathrm{GHz}$ (dashed red curve) caused by the fundamental half-wavelength resonance of a capacitively-loaded dipole, see Fig. 2(b), where surface currents are plotted. Note that the vertical arms act as capacitive loading of the horizontal arm. When two layers are stacked, a resonance hybridization occurs due to the magnetic coupling between layers [Fig. 2(c)] introducing two dips in the spectrum [Fig. 2(a), solid red curve]. Beyond the second dip, almost full transmission is achieved. The mechanism is similar under vertical polarization. In this case, for the single layer, the drop happens at $115.6 \mathrm{GHz}$ (dashed blue curve), caused again by the fundamental dipole resonance [Fig. 2(d)]. When two layers are stacked, this resonance undergoes hybridization due to electric coupling [Fig. 2(e)], and the lowest frequency dip takes place near $90 \mathrm{GHz}$, which is preceded by a peak of transmission. The resonance proximity generates a fast phase change that leads to a phase difference between vertical and horizontal polarization near $180^{\circ}$ at 86.2 GHz. At this frequency, amplitude values for $T_{x x}$ and $T_{y y}$ are both around $-0.55 \mathrm{~dB}$ and the absorbance of the structure (due to metallic and dielectric losses) is around $-12.2 \mathrm{~dB}$ Such a phase difference fulfills the conditions needed for an HWP since transmission is maximum for both components near that frequency.

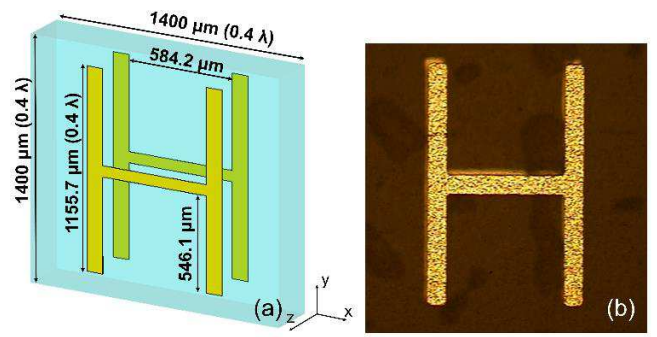

Fig. 1. (a) Schematic view of the H-shaped bi-layer unit cell with the main dimensions. The metallic areas and dielectric substrate are represented in yellow and blue, respectively. (b) Microphotograph of a unit cell of the fabricated metalens.

Finally, the transmission and reflection coefficients under left-handed circularly polarized (LHCP) excitation are shown in the decibel scale in Fig. 2(f). From this figure, a cross-polar transmission coefficient $\left(T_{R L}\right)$ around $-0.9 \mathrm{~dB}$ is obtained at $86.2 \mathrm{GHz}$, demonstrating a high transmission efficiency $(90.15 \%)$ of right-handed circular polarization (RHCP). At the same frequency, the reflection coefficients (both $R_{L L}$ and $R_{R L}$ ) and the transmitted co-polar component $\left(T_{L L}\right)$ are below $-10 \mathrm{~dB}$, indicating a good matching and a proper rejection of the non-desired polarization. Moreover, the cross-polar discrimination (also called extinction ratio) at the output, defined as XPD $=T_{R L} / T_{L L}$ is above $10 \mathrm{~dB}$ within a fractional bandwidth of $3.4 \%$, showing the highest value at 87.7 $\mathrm{GHz}$, where the phase difference between linear 
components is precisely $180^{\circ}$. Therefore, based on the previous results, the design frequency is fixed at $87 \mathrm{GHz}$ as a trade-off between maximum $\mathrm{T}_{\mathrm{RL}}$ and XPD.

In the next step, to achieve the spatial wavefront shaping and, therefore, implement the PB metalens, we calculated the required spatial phase profile $\varphi(x, y)$ of the metasurface [made of $63 \times 63$ meta-atoms (unit cells)] using Eq. (1):

$$
\varphi(x, y)=\frac{2 \pi f_{0}}{c_{0}}\left(\sqrt{x^{2}+y^{2}+F L^{2}}-F L\right)
$$

where the design frequency is fixed at $f_{0}=87 \mathrm{GHz}$ $\left(\lambda_{0}=3.45 \mathrm{~mm}\right)$, the focal length at $F L=70 \mathrm{~mm}$, and $c_{0}$ is the speed of light in a vacuum. The calculated ideal spatial phase profile $\varphi(x, y)$ is depicted in Fig. 3(a).
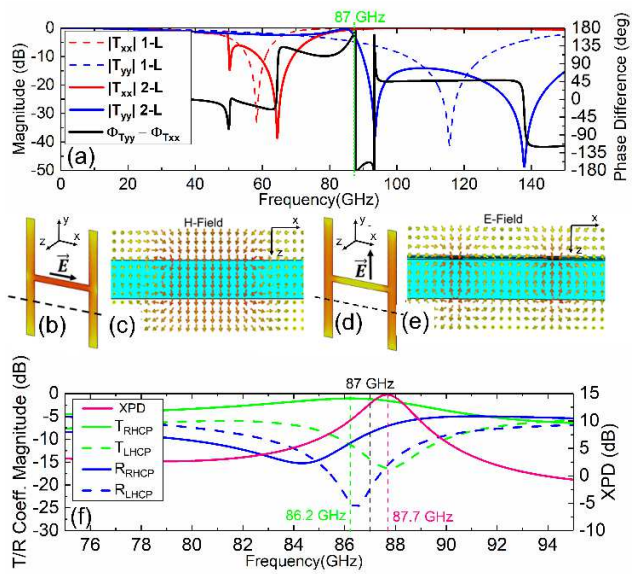

Fig. 2. (a) Unit cell transmission coefficient magnitude for one layer (dashed) and two layers (solid) calculated using linear vertical (blue) and horizontal (red) polarization excitation. Transmission coefficient phase difference between vertical and horizontal polarization for two layers (black curve). (b), (d) Snapshot of the surface currents maximum magnitude for one layer at the dip for $x$ and $y$-polarized incident wave, $58.4 \mathrm{GHz}$ and $115.7 \mathrm{GHz}$, respectively. (c), (e) Snapshot of the magnetic/electric field lines between the bi-layer design at the cutting plane (black dashed lines) depicted in (b) and (d) at $87 \mathrm{GHz}$. (f) Unit cell transmission (green) and reflection (blue) coefficients magnitude in $\mathrm{dB}$ when the structure is excited by an LHCP plane wave at normal incidence. Solid/dashed lines correspond to RHCP/LHCP polarization. The solid magenta line represents the XPD value at the output.

According to the PB phase principle, the rotation angles for every unit cell of the PB metalens correspond with half of the desired ideal phase shift at each surface position $\varphi(x, y)$ The implemented phases are depicted in Fig. 3(b), almost perfectly coinciding with the ideal values, but not precisely, as can be appreciated in Fig. 3(c). These slight discrepancies are probably due to a minor mismatching introduced in some rotation cases. Although the resonators were designed to never invade adjacent cells for any rotation angle, the coupling between them can differ depending on the angle, modifying the expected phase at the output. Thus, a correction in these cases has been applied, selecting the rotation angle among the simulated ones that provides the most approximated value to the desired phase shift but slightly different from the ideal one. The focal performance of the device was evaluated using a semi-analytical HuygensFresnel (H-F) method ${ }^{27}$ and numerical simulations.

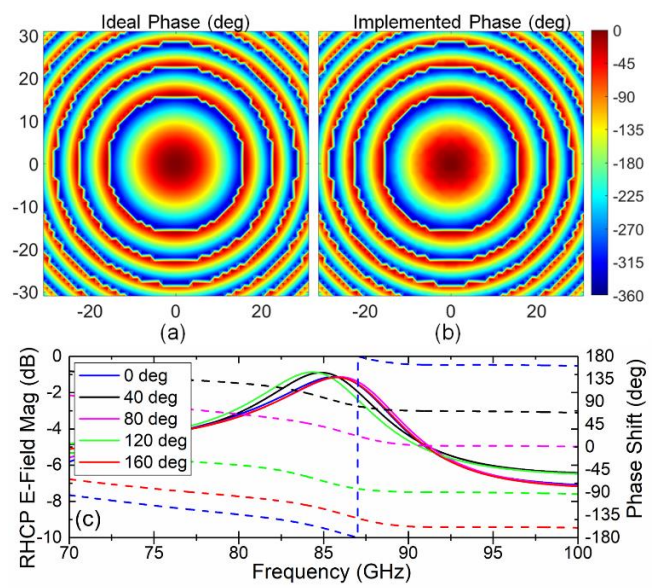

Fig. 3. Spatial phase profile at the $\mathrm{PB}$ metalens $(63 \times 63$ meta-atoms $)$ as a function of the meta-atoms position $(x, y)$ for the ideal (a) and implemented (b) metalens at $87 \mathrm{GHz}$. (c) RHCP E-Field magnitude (solid lines) and phase (dashed lines) for unit cell rotation angles of $0^{\circ}, 40^{\circ}, 80^{\circ}, 120^{\circ}$ and $160^{\circ}$.

The fabricated PB metalens is depicted in Fig. 4(a). The set-up used in the experimental characterization is shown in Fig. 4(b). It consists of a septum polarizer fed transmitting corrugated horn antenna with a low axial ratio at the frequencies of interest, which delivers LHCP polarization over the lens. A linearly polarized open-ended rectangular waveguide probe (WR-10 standard waveguide) with sharp edges to reduce scattering was placed in the optical axis ( $z$ axis) to act as a receiver. It must be taken into account that with this configuration there is a systematic phase error between linear polarization components in reception due to the $90^{\circ}$ rotation of the probe to obtain both the $x$ - and $y$-axis components. This rotation produces a $z$-shift of the probe of $400 \mu \mathrm{m}$ approximately (estimated from previous measurements with the same set-up), giving rise to a phase error between linear components of $41.5^{\circ}$ at $87 \mathrm{GHz}$ This systematic error is corrected in the post-processing to obtain the circular components. Both the transmitting antenna and the probe datasheets can be found in Anteral S.L. webpage ${ }^{28}$. An exhaustive alignment of the set-up components was carried out using a laser tracker, and a preliminary calibration was taken before placing the metalens. The measurements were obtained by moving the probe position along the $z$-axis from 62 to $112 \mathrm{~mm}$ with a $2 \mathrm{~mm}$ step. The field distribution 
was raster-scanned at each position in the $x y$-plane from -6 $\mathrm{mm}$ to $6 \mathrm{~mm}$ with a $0.5 \mathrm{~mm}$ step $(25 \times 25$ points in total). The frequency was swept from 75 to $105 \mathrm{GHz}$ with a $0.2 \mathrm{GHz}$ step at each location. This procedure was repeated for both horizontal and vertical orientations of the probe, which measured both polarizations. Final post-processing was done to compose the measured results from a linear to circular basis.

The H-F analysis and simulation results are shown in the first two columns of Fig. 5, respectively. In that figure, panels $[(\mathrm{a}),(\mathrm{b})],[(\mathrm{d}),(\mathrm{e})]$ and $[(\mathrm{g}),(\mathrm{h})]$ represent the normalized RHCP electric field magnitude in the $x z-, y z$ - and $x y$-cutting planes, respectively. In general, the agreement between analytical and numerical results is good (see Table I).

The numerical analysis of the device's full 3D model (with a size of $63 \times 63$ unit cells) was carried out using the time domain solver of CST Studio Suite ${ }^{\circledR}$, exciting the structure with a normally incident LHCP plane wave and using open add space as boundary conditions. The calculation domain was extended along the $z$-direction up to $90 \mathrm{~mm}$ to visualize the focal point. The field distribution was obtained at $87 \mathrm{GHz}$ by defining a CST Studio Suite built-in E-Field monitor.

The minor disagreements between them can be attributed to the coupling between adjacent cells (disregarded in the $\mathrm{H}$ $\mathrm{F}$ analysis) and point source approximation in $\mathrm{H}-\mathrm{F}$ analysis. The full-width at half-maximum (FWHM) is identical for the $x$ - and $y$-axis in simulation and $\mathrm{H}-\mathrm{F}$, ensuring a perfect circularity of the focus. The simulated enhancement (defined as the ratio between the power at the focus with and without the lens) is $16.9 \mathrm{~dB}$ and $15.2 \mathrm{~dB}$ using $\mathrm{H}-\mathrm{F}$ analysis. The simulated FWHM is $0.8 \lambda_{0}$, a bit smaller than the Rayleigh diffraction limit $\left(0.61 \lambda_{0} / \mathrm{NA}=0.87 \lambda_{0}\right)$, where $\mathrm{NA}$ is the numerical aperture of the lens. This limit is usually employed to evaluate the resolution of a lens, i.e., the minimum distance between two point sources that the lens can resolve. Furthermore, the simulated focus presents a low sidelobe level of $0.21(-13.26 \mathrm{~dB})$ at $\pm 4.6 \mathrm{~mm}$, as shown in Fig. 5(f).

In Fig. 5(c) (dashed green curve), the experimental FL appears at $94 \mathrm{~mm}$ (see Table I), which deviates from the numerical value by $27 \mathrm{~mm}\left(7.8 \lambda_{0}\right)$. Likewise, the depth of focus (DOF) is wider than in the simulation. We estimate a broadening of $13.9 \mathrm{~mm}\left(4 \lambda_{0}\right)$ since it is impossible to measure the actual value due to the $z$-axis stage's limitations (positioner could move along $z$ in the range 62 to $112 \mathrm{~mm}$ from the metasurface due to the mechanical restrictions). Thus, this estimated value is calculated as double the width measured between the point for the maximum E-Field value and the point where the E-Field decays by $3 \mathrm{~dB}(0.7)$, which happens at $80 \mathrm{~mm}$. Furthermore, by looking at the normalized level of $T_{L L}$ at the focus, an experimental XPD value of 25 $(27.9 \mathrm{~dB})$ is observed, which is even higher than the simulation value equal to $12(21.6 \mathrm{~dB})$, demonstrating an outstanding level of polarization conversion at the focus.

The FWHM values for simulation and measurement, depicted in Fig. 5(f), also show that the experimental focus undergoes a widening in the $x$ - and $y$-axis of $1.4 \mathrm{~mm}\left(0.4 \lambda_{0}\right)$, so the experimental lens resolution is $1.2 \lambda_{0}(\approx 7 \%$ above of the Rayleigh resolution limit).
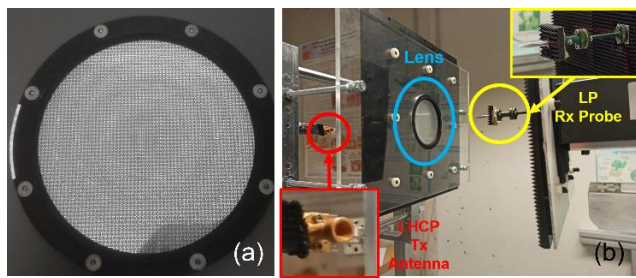

TABLE I

COMPARISON H-F SIMULATION AND EXPERIMENTAL

\begin{tabular}{ccccc}
\hline \hline Model & $\begin{array}{c}F W H M \\
(\mathrm{~mm})\end{array}$ & $\begin{array}{c}{ }^{*} \mathrm{DOF} \\
(\mathrm{mm})\end{array}$ & $\begin{array}{c}{ }^{* *} \mathrm{FL} \\
(\mathrm{mm})\end{array}$ & $\begin{array}{c}{ }^{* * *} \mathrm{Enh} \\
(\mathrm{dB})\end{array}$ \\
\hline$H-F$ & 2 & 10.5 & 69 & 15.2 \\
Simulation & 2.7 & 14.1 & 67 & 16.9 \\
Experimental & 4.1 & 28 & 94 & 19.7 \\
\hline \hline
\end{tabular}

${ }^{*}$ DOF is the depth of focus

${ }^{* *} F L$ is the focal length

${ }^{* * *}$ Enh. is the enhancement

Fig. 4. (a) General photograph of the fabricated metalens (diameter of $87 \mathrm{~mm}$ ) mounted in a circular holder. (b) Experimental set-up with the main elements highlighted: Transmitting antenna (red), metalens (blue), and receiving probe (yellow). The bottom left, and top right insets show detailed views of the transmitting antenna and receiving probe. The metalens and receiving probe are surrounded by absorbent material (black tiles).
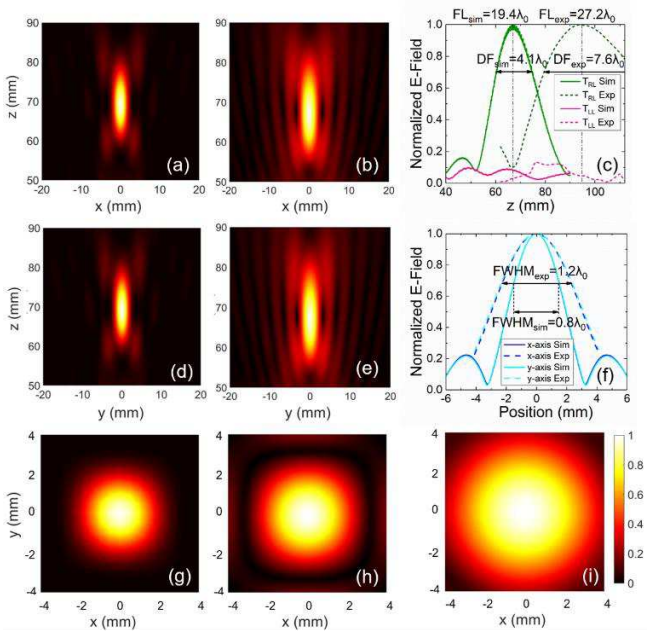

Fig. 5. Normalized RHCP E-field magnitude at $87 \mathrm{GHz}$ extracted from $\mathrm{H}-\mathrm{F}$ analysis and simulation for $x z$-plane (a),(b), $y z$-plane (d),(e) and $x y$-plane (g),(h). (c) Simulated and measured normalized E-field magnitude for RHCP and LHCP along the optical axis ( $z$ axis). (f) Simulated and measured normalized E-field magnitude for $\mathrm{RHCP}$ at the focus tracked along $x$ - and $y$-axes. In (i) the normalized RHCP E-field magnitude at $x y$-plane of the experimental metalens is depicted at $87 \mathrm{GHz}$.

This broadening of the focal spot in the measurement is probably due to the point spread function of the detecting 
probe since this is not a point detector. The FWHM widening can be observed by comparing Figs. 5(g-i), where the H-F, numerical, and the experimental $x y$-planes, respectively, are shown. Moreover, all cases demonstrate the circularity of the focus. Finally, the observed maximum power enhancement is $19.7 \mathrm{~dB}$.

The discrepancies between simulation and measurement can be ascribed to the non-uniform phase distribution provided by the transmission antenna on the lens' plane in the experimental set-up. The transmitting horn antenna is located at $21 \mathrm{~cm}$ from the metasurface lens's plane, ensuring that the maximum amplitude difference between the center and the edges of the metalens is of only $1.6 \mathrm{~dB}$. Nevertheless, we found that the phase distribution on the metalens plane is not uniform, introducing additional phases that disturb the final focal point position. Unfortunately, this distance is the current maximum value allowed by our mechanical instrumentation in transmission. A further semi-analytical study (not shown here) has confirmed this disagreement in the FL (using the H-F method and introducing additional phases in each metalens' unit cell) although a deeper study is needed to fit quantitatively the experimental results. This study is being performed and it will be presented in a subsequent paper. Nevertheless, despite this disagreement due to the non-uniform phase illumination, it can be affirmed that the PB metalens has an excellent behavior as a polarization converter and a lens simultaneously, reaching a very high level of XPD.

In summary, we have analytically, numerically, and experimentally demonstrated an ultrathin $(<\lambda / 13)$ PBM lens. The structure operates at $87 \mathrm{GHz}$ and converts the handedness of the $\mathrm{CP}$ incident waves reaching a transmission efficiency of $90.15 \%$ with only two layers. Furthermore, a very high level of XPD $(27.9 \mathrm{~dB})$ at the focus is presented, which indicates an outstanding level of polarization conversion and almost perfect circular polarization at the focal point. The energy of the incident wave is focused at 27.2 $\lambda$ from the metasurface and presents a DOF around $7 \lambda$ with an enhancement of $19.7 \mathrm{~dB}$ and an FWHM near $\lambda$. The advantages provided by these ultra-thin PB metalens are the very high cross-polar transmission efficiency and the excellent rejection of co-polarization, allowing highly pure circular cross-polarization. Hence, the proposed device may find application in lens systems operating at millimeter waves where ease of integration is essential, and circular polarization conversion with high efficiency and purity are required.

\section{ACKNOWLEDGEMENTS}

This research was funded by Spanish Ministerio de Ciencia, Innovación y Universidades, Project RTI2018-094475-B-I00 (MCIU/AEI/FEDER,UE). Sergei Kuznetsov acknowledges support from the Ministry of Science and Higher Education of the Russian Federation, Project 075-15-2020-797.

\section{DATA AVAILABILITY}

The data that support the findings of this study are available from the corresponding author upon reasonable request.

\section{REFERENCES}

${ }^{1}$ S.B. Glybovski, S.A. Tretyakov, P.A. Belov, Y.S. Kivshar, and C.R. Simovski, Phys. Rep. 634, 1 (2016).

2 J. Jung, H. Park, J. Park, T. Chang, and J. Shin, Nanophotonics 9, 3165 (2020).

${ }^{3}$ K. Achouri and C. Caloz, Nanophotonics 7, 1095 (2018).

${ }^{4}$ X.-P. Dong, J.-R. Cheng, F. Fan, S.-T. Xu, X.-H. Wang, and S.-J. Chang, Opt. Express 27, 202 (2019).

${ }^{5}$ J. Zi, Y. Li, X. Feng, Q. Xu, H. Liu, X.-X. Zhang, J. Han, and W. Zhang, Phys. Rev. Appl. 13, 034042 (2020).

${ }^{6}$ W. Mo, X. Wei, K. Wang, Y. Li, and J. Liu, Opt. Express 24, 13621 (2016).

${ }^{7}$ F. Ding, Z. Wang, S. He, V.M. Shalaev, and A. V. Kildishev, ACS Nano 9, 4111 (2015)

${ }^{8}$ E. Hasman, G. Biener, A. Niv, and V. Kleiner, in Prog. Opt. (Elsevier Masson SAS, 2005), pp. 215-289.

${ }^{9}$ L. Shao-he, L. Jiu-sheng, and S. Jian-zhong, Opt. Mater. Express 9, 1118 (2019).

${ }^{10}$ M.I. Khan and F.A. Tahir, J. Phys. D. Appl. Phys. 50, 43LT04 (2017).

${ }^{11}$ A. Moreno-Penarrubia, S.A. Kuznetsov, and M. Beruete, IEEE Trans. Antennas Propag. 68, 7700 (2020).

${ }^{12}$ W.T. Chen, A.Y. Zhu, and F. Capasso, Nat. Rev. Mater. 5, 604 (2020).

${ }^{13}$ Y. Nakata, Y. Taira, T. Nakanishi, and F. Miyamaru, Opt. Express 25, 2107 (2017).

${ }^{14}$ M. Jia, Z. Wang, H. Li, X. Wang, W. Luo, S. Sun, Y Zhang, Q. He, and L. Zhou, Light Sci. Appl. 8, 16 (2019).

15 C.-C. Chang, D. Headland, D. Abbott, W. Withayachumnankul, and H.-T. Chen, Opt. Lett. 42, 1867 (2017).

${ }^{16}$ H.-X. Xu, H. Liu, X. Ling, Y. Sun, and F. Yuan, IEEE Trans. Antennas Propag. 65, 7378 (2017).

17 M.R. Akram, M.Q. Mehmood, X. Bai, R. Jin, M Premaratne, and W. Zhu, Adv. Opt. Mater. 7, 1801628 (2019).

${ }^{18}$ W. Luo, S. Sun, H.-X. Xu, Q. He, and L. Zhou, Phys. Rev Appl. 7, 044033 (2017)

${ }^{19}$ J. Duan, H. Guo, S. Dong, T. Cai, W. Luo, Z. Liang, Q. He, L. Zhou, and S. Sun, Sci. Rep. 7, 1354 (2017).

${ }^{20}$ W.S.L. Lee, S. Nirantar, D. Headland, M. Bhaskaran, S. Sriram, C. Fumeaux, and W. Withayachumnankul, Adv. Opt. Mater. 6, 1700852 (2018).

${ }^{21}$ L. Wang, S. Liu, X. Kong, Y. Wen, and X. Liu, Electron. Lett. 55, 1168 (2019).

${ }^{22}$ S. Jiang, C. Chen, H. Zhang, and W. Chen, Opt. Express 26, 6466 (2018).

${ }^{23}$ X. Ding, F. Monticone, K. Zhang, L. Zhang, D. Gao, S.N. Burokur, A. de Lustrac, Q. Wu, C.-W. Qiu, and A. Alù, Adv. Mater. 27, 1195 (2015).

${ }^{24}$ A. Arbabi and A. Faraon, Sci. Rep. 7, 43722 (2017).

${ }^{25}$ R. Mao, G. Wang, T. Cai, K. Liu, D. Wang, and B. Wu, Opt. Express 28, 31216 (2020).

${ }^{26}$ S.A. Kuznetsov, A.V. Arzhannikov, V.V. Kubarev, P.V. Kalinin, M. Sorolla, M. Navarro-Cia, M. Aznabet, M Beruete, F. Falcone, Y.G. Goncharov, B.P. Gorshunov, A.V. Gelfand, and N.I. Fedorinina, Key Eng. Mater. 437, 276 
(2010).

27 B. Orazbayev, V. Pacheco-Peña, M. Beruete, and M.

Navarro-Cía, Opt. Express 23, 8555 (2015).

${ }^{28}$ www.anteral.com. 


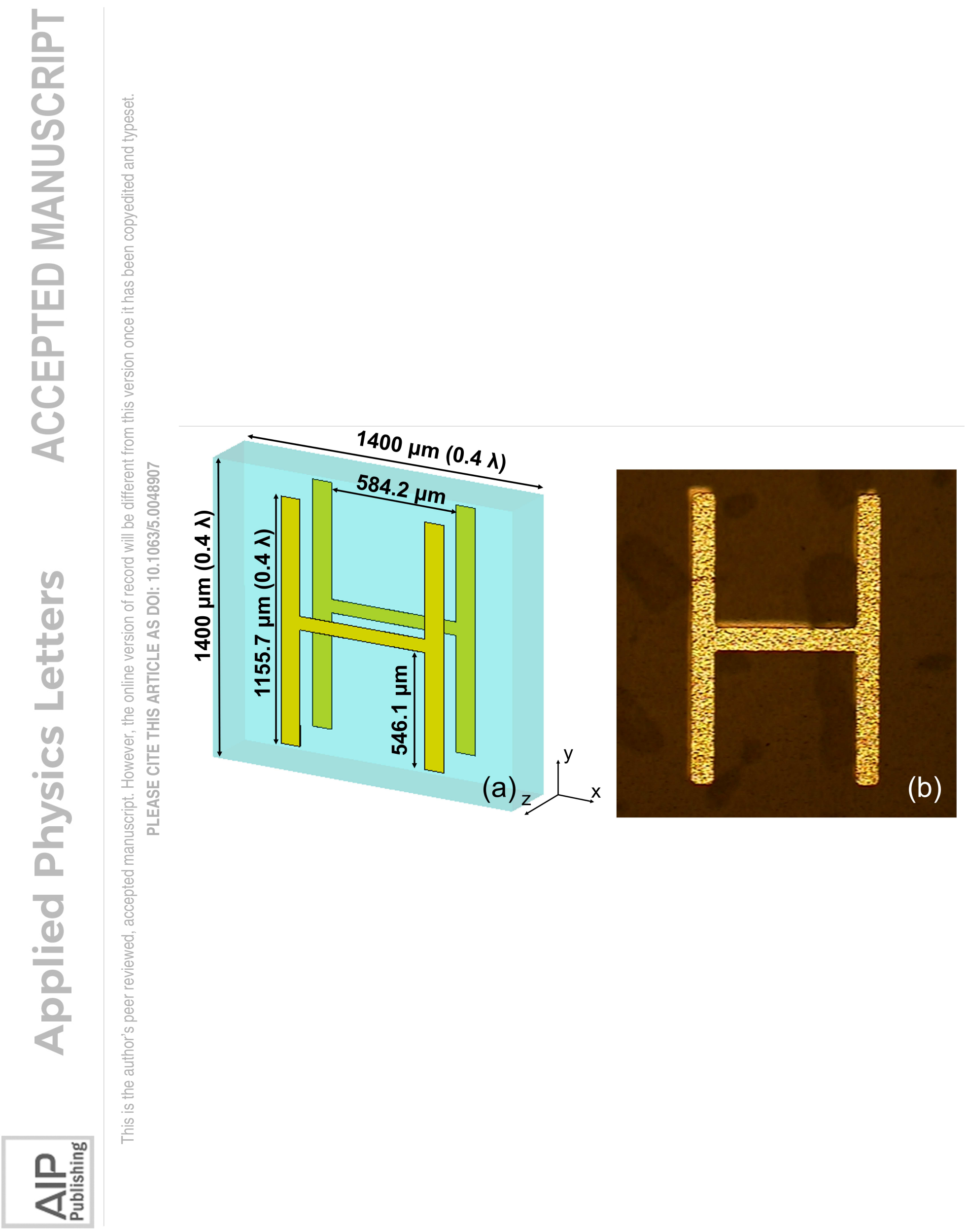



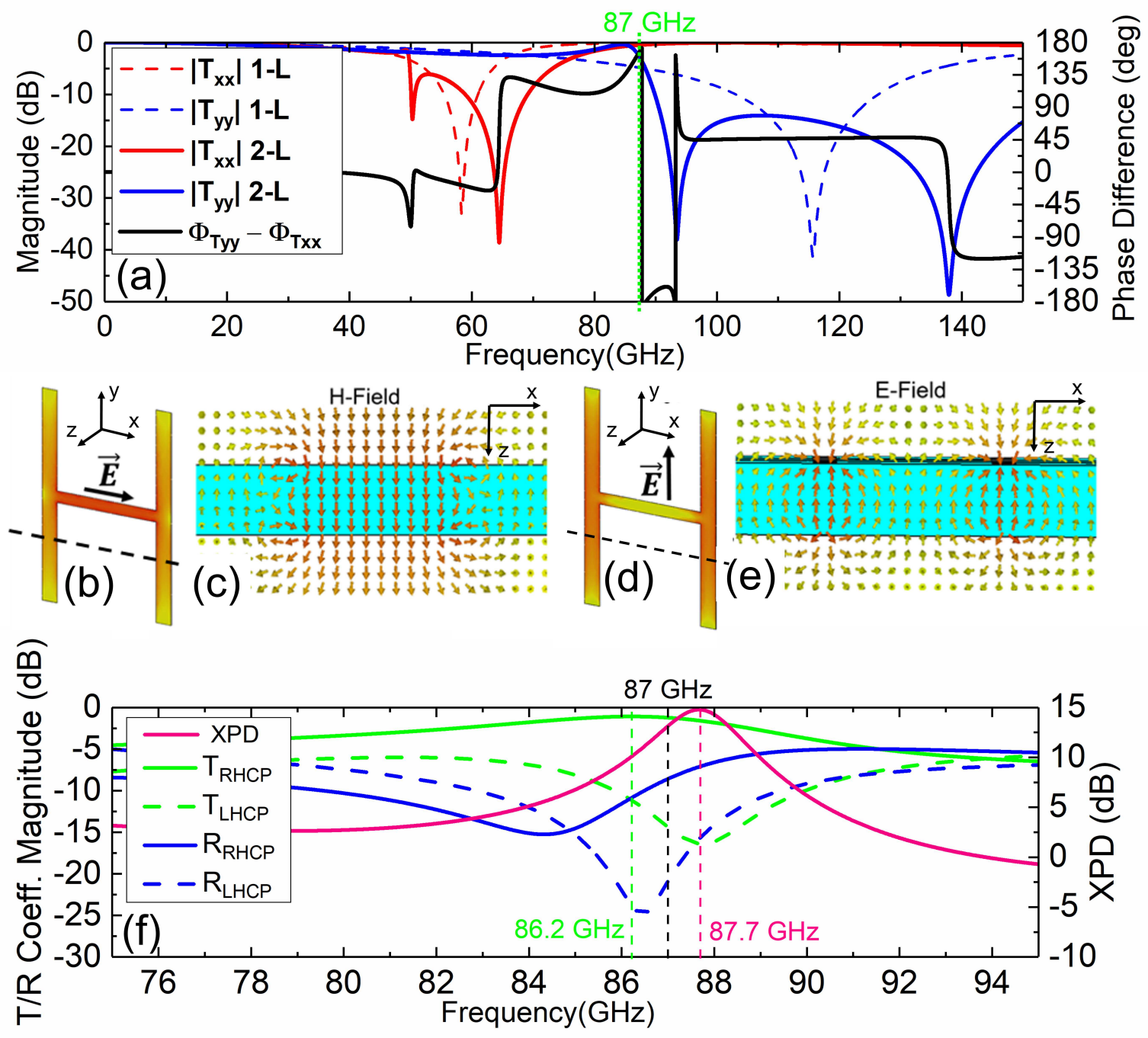


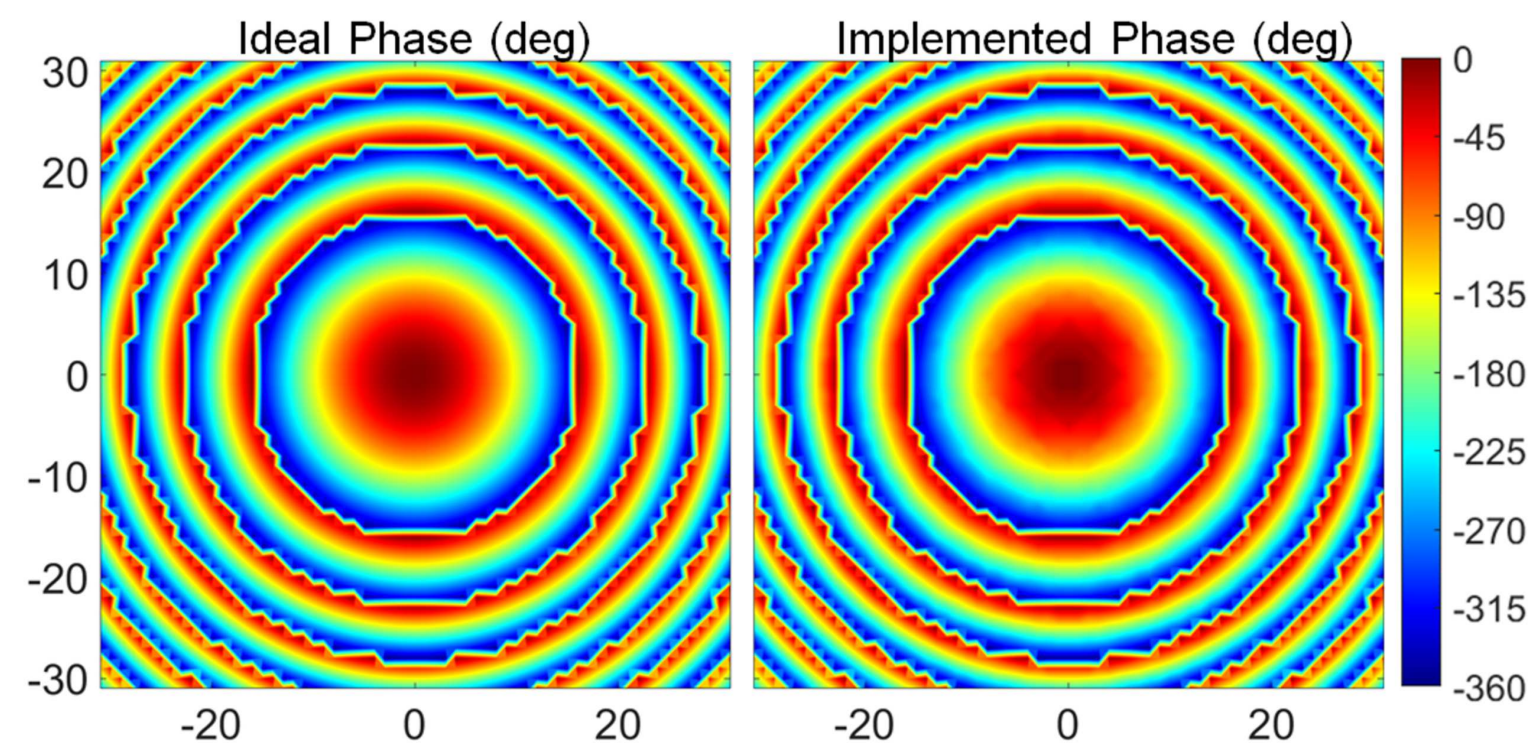

(a)

(b)

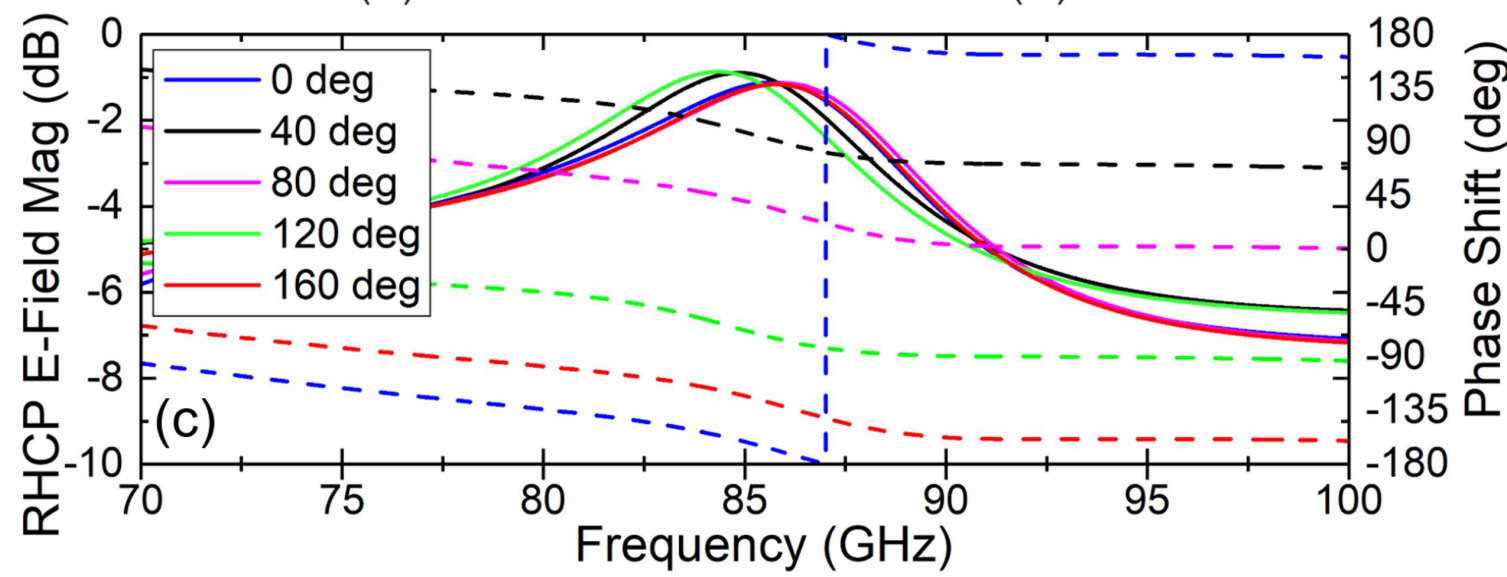




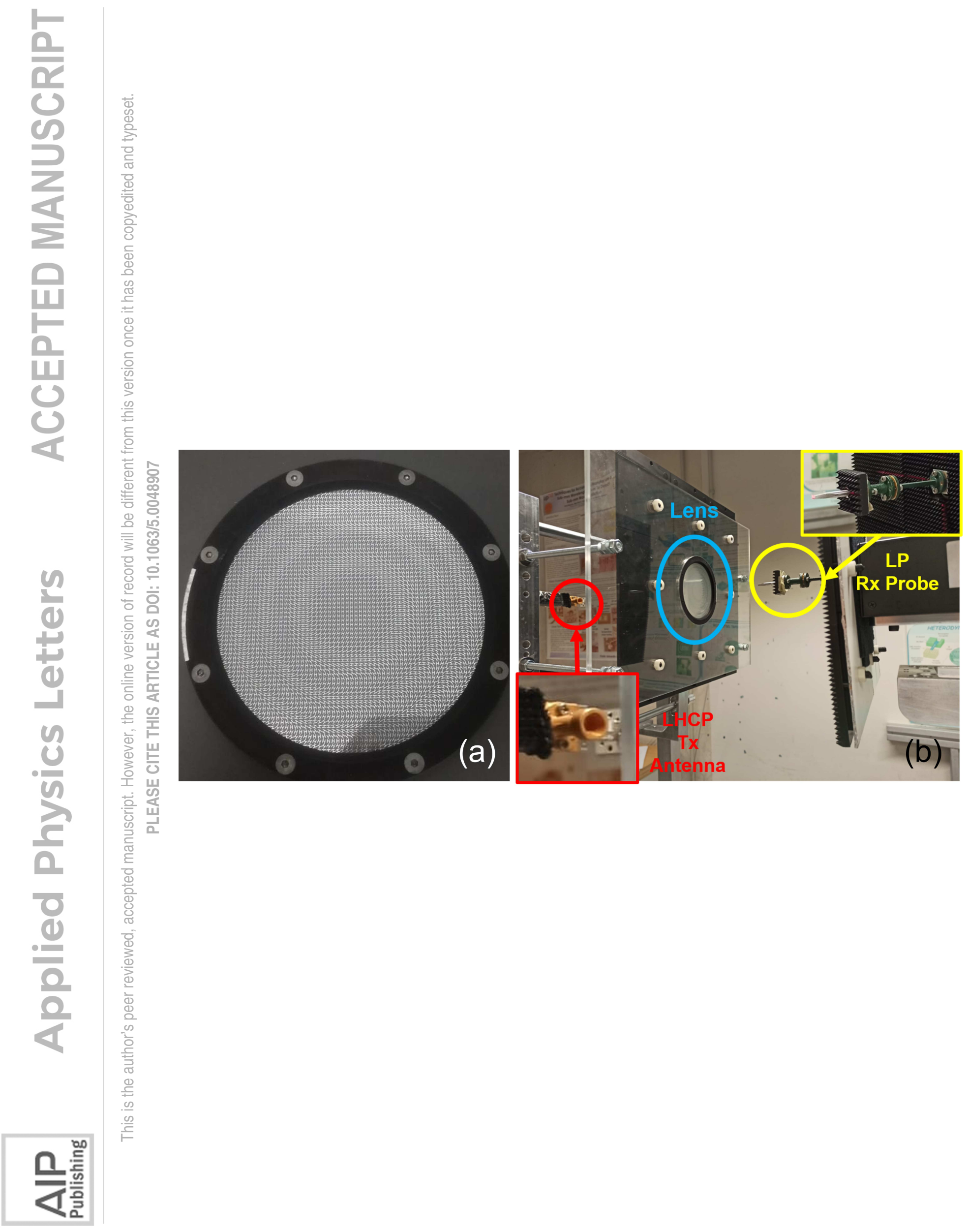



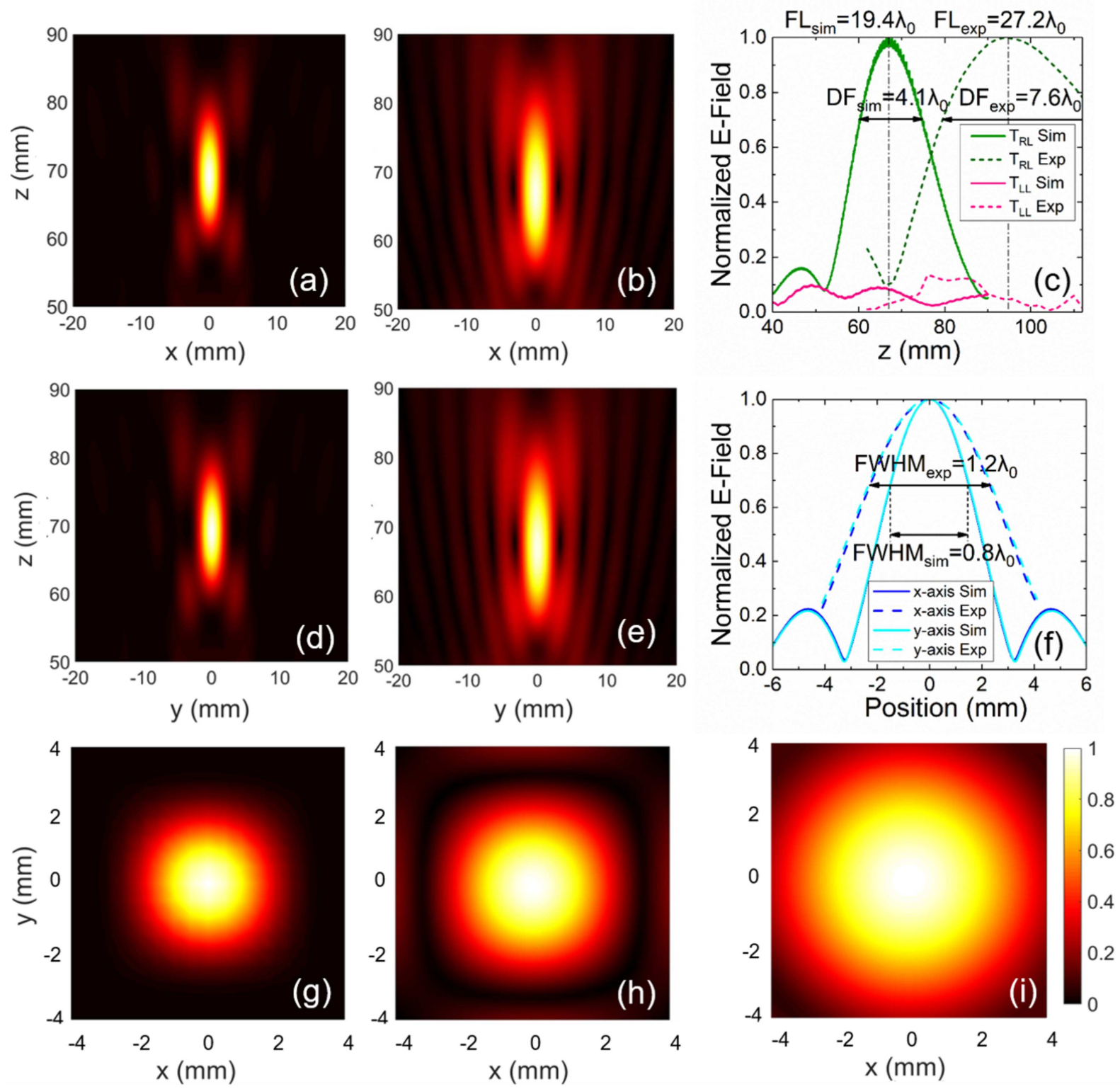